\title{
Reduction of truncation errors in planar near-field aperture antenna measurements using the method of alternating orthogonal projections
}

Martini, Enrica; Breinbjerg, Olav; Maci, Stefano

Published in:

Proceedings of the European Conference on Antennas and Propagation (EuCAP)

Link to article, DOI:

10.1109/EUCAP.2006.4584841

Publication date:

2006

Document Version

Publisher's PDF, also known as Version of record

Link back to DTU Orbit

Citation (APA):

Martini, E., Breinbjerg, O., \& Maci, S. (2006). Reduction of truncation errors in planar near-field aperture antenna measurements using the method of alternating orthogonal projections. In Proceedings of the European Conference on Antennas and Propagation (EUCAP) (pp. 1-6). IEEE.

https://doi.org/10.1109/EUCAP.2006.4584841

\section{General rights}

Copyright and moral rights for the publications made accessible in the public portal are retained by the authors and/or other copyright owners and it is a condition of accessing publications that users recognise and abide by the legal requirements associated with these rights.

- Users may download and print one copy of any publication from the public portal for the purpose of private study or research.

- You may not further distribute the material or use it for any profit-making activity or commercial gain

- You may freely distribute the URL identifying the publication in the public portal 


\title{
REDUCTION OF TRUNCATION ERRORS IN PLANAR NEAR-FIELD APERTURE ANTENNA MEASUREMENTS USING THE METHOD OF ALTERNATING ORTHOGONAL PROJECTIONS
}

\author{
Enrica Martini ${ }^{1}$, Olav Breinbjerg ${ }^{1}$, and Stefano Maci $^{2}$ \\ ${ }^{1}$ Ørsted.DTU, Electromagnetic Systems, Technical University of Denmark, 2800 Lyngby, Denmark, Email: \\ ema@oersted.dtu.dk, ob@oersted.dtu.dk \\ ${ }^{2}$ Department of Information Engineering, University of Siena, Siena, 53100, Italy, Email: macis@ dii.unisi.it
}

\begin{abstract}
A simple and effective procedure for the reduction of truncation error in planar near-field to far-field transformations is presented. The starting point is the consideration that the actual scan plane truncation implies a reliability of the reconstructed plane wave spectrum of the field radiated by the antenna only within a certain region inside the visible range. Then, the truncation error is reduced by a Maxwellian continuation of the reliable portion of the spectrum: after back propagating the measured field to the antenna plane, a condition of spatial concentration of the primary field is exploited to define a convergent iterative process which is also stable against moderately noisy data. Far-field patterns reconstructed from both simulated and measured near-field data demonstrate the effectiveness of the proposed procedure.
\end{abstract}

\section{INTRODUCTION}

Planar near-field measurements constitute a well known and widely used technique for characterizing the radiation from antennas $[1,2]$. However, the finite size of the scanning area introduces an error in the reconstructed farfield pattern, denoted in literature as truncation error. Due to this error, the reconstructed radiation pattern is reliable only within a certain angular region. With a physical equivalence, the truncation error may be attributed to the presence of a perfectly absorbing ("black") screen positioned around the scan plane. Thus, the angular region of reliability of the reconstructed pattern is bounded by the rays running from the edge of the antenna aperture through the boundary of the scan area [3]. This imposes a minimum size of the scan plane for a desired angular region of coverage and a given separation distance between the antenna under test (AUT) and the measurement plane (a minimum distance is necessary to reduce multiple reflections). However, long measurement times and complex facilities are required to cover large scan areas. A method allowing one to reduce truncation errors without requiring additional measurements would significantly speed up and simplify the measurement process. Truncation effects can be partially mitigated by applying to the near-field scan data proper window functions having a smooth taper at the edges of the scan plane $[4,5,6]$. However, only narrow regions of the far-field pattern can be corrected using this technique. An alternative approach consists in employing near-field data to determine equivalent magnetic currents on the AUT aperture through the solution of an EFIE [7] or an optimization technique [8]. However, these methods may become highly time-consuming for electrically large apertures. A different strategy based on a priori information on the AUT geometry, exploits a nonreduntant representation of the radiated field to estimate the near-field data falling outside the scanning area [9]. A procedure based on the method of minimum extension to extrapolate antenna measurements data when the current distribution can be represented as a system of point sources and line sources has also been presented [10]. A key point emerging from most of the cited works is that the utilization of available a priori information is essential to define an efficient pattern reconstruction process.

The purpose of this paper is to present a new simple and effective procedure for the reduction of truncation error in plane-rectangular near-field measurements of aperture antennas.

The basic idea of the proposed algorithm is to extrapolate the radiated pattern outside the reliable region exploiting the hypothesis that, on the AUT plane, the electric field is mostly concentrated inside the antenna aperture. To this end, the simple relationship existing between the far-field pattern and the visible part of the Plane Wave Spectrum (PWS) of the aperture field [11] is exploited to argue that the aperture PWS reconstructed from truncated measurement is reliable only inside a certain spectral region. As a consequence, the problem of pattern reconstruction is restated in terms of extrapolating the remaining part of the visible region of the PWS from its reliable portion. A mathematically equivalent problem has previously been investigated in the framework of image superresolution, aiming at increasing the resolution of images [12]. Any $a$ priori information which imposes appropriate constraints on the function to be restored is necessary to avoid illposedness of the problem. In the case of image processing, examples of possible constraints are so-called nonnegativity, level and edge preserving constraints. Furthermore, for finite objects, a space constraint resulting from 
their known physical extension can be used. This condition can be assumed also in the case of near-field planar measurements of aperture antennas after back propagating the field to the AUT plane, as long as the field can be assumed concentrated on the antenna aperture. Under this hypothesis, a rigorous spectrum extrapolation is based on the analytic continuation of entire spectral functions; it is indeed well known that a function with finite spatial extent possesses a spectrum which is an entire function, i.e. an analytic function on the entire complex plane. The extrapolation process is however delicate in presence of noise. Indeed, the conventional procedure of analytic continuation based on calculating local Taylor converging series can not be applied to measured data, since the derives needed are very noise sensitive; furthermore, the Taylor series must be truncated in practice, thus introducing a further source of error. An alternative approach consists in expanding the given portion of the spectrum in terms of prolate spheroidal wave functions [13], to obtain a representation valid everywhere [14]. However, also this method is plagued by noise amplification [15].

In this paper, the spectral extrapolation is performed by utilizing the general method of alternating orthogonal projections. Such a procedure was first proposed by Gerchberg [12] in the framework of image resolution enhancement, and further investigated by Papoulis [16]. It has been demonstrated that, in the absence of noise, the iterative algorithm converges in norm to the correct solution, and it is also quite robust against moderately noisy data. As a consequence, this method can provide also a good estimate of the wide angle lobes of the far-field pattern, when applied to the problem of pattern reconstruction.

Since the proposed technique relies on the assumption that the primary field is concentrated inside the AUT aperture, it is particularly suitable to the analysis of radiation of apertures in large ground planes; however, numerical results based on simulated as well as measured near-field data have shown that it can provide a significant reduction of truncation error for a much wider class of aperture antennas.

It is worth noting that the method described in this paper also provides an accurate representation of the aperture field distribution. In fact, a similar iterative procedure has already been applied to determine, from far-field data, surface distortions in large reflector antennas [17] and the individual element excitations of planar arrays for pattern synthesis [18] or array antenna diagnosis and calibration [19].

This contribution is organized as follows: first, the mathematical formulation of the method of alternating orthogonal projections is briefly summarized in Section 2; then, its application to the problem of pattern reconstruction is described in Section 3. Numerical results are presented in Section 4. Finally, conclusions are drawn in Section 5.

\section{THE METHOD OF ALTERNATING ORTHOG- ONAL PROJECTIONS}

The method of alternating orthogonal projections [20] is a general iterative algorithm that can be used to effectively reconstruct a partially known signal starting from the available data and other prior knowledge. The mathematical formulation of the method is summarized in the following.

Consider a Hilbert space $\mathscr{H}$ with elements $f, p, q$. Let $\mathscr{P}$ be any closed linear subspace of $\mathscr{H}$ and $\mathscr{Q}$ its orthogonal complement, that is the set containing all the elements of $\mathscr{H}$ which are orthogonal to every element in $\mathscr{P}$. According to the projection theorem, every $f \in \mathscr{H}$ possesses a unique decomposition

$$
f=p+q
$$

in which $p \in \mathscr{P}$ and $q \in \mathscr{Q}$. The linear operators $P$ and $Q$ are then introduced to define the projections of a function $f$ onto $\mathscr{P}$ and $\mathscr{Q}$, respectively, i.e.

$$
\begin{aligned}
& p=P f ; \\
& q=Q f=(f-P f)=(1-P) f .
\end{aligned}
$$

Now, assume that $f$ belongs to a closed linear subspace $\mathscr{P}_{1}$, and that only its projection onto another closed linear subspace $\mathscr{P}_{2}, p_{2}=P_{2} f$, is available. Then, it can be demonstrated [20] that $f$ is uniquely determined by $p_{2}$ if and only if the two sets $\mathscr{P}_{1}$ and $\mathscr{Q}_{2}$ have empty intersection. In that case, in the absence of noise, there exists an effective recursive algorithm for the recovery of $f$ employing only the operations of projection onto $\mathscr{P}_{1}$ and $\mathscr{Q}_{2}$ Indeed, the condition $f \in \mathscr{P}_{1}$ implies $P_{1} f=f$, hence, the following relationship is readily derived

$$
p_{2}=P_{2} f=P_{2} P_{1} f=\left(1-Q_{2}\right) P_{1} f=f-Q_{2} P_{1} f .
$$

Starting from there, the following sequence is generated

$$
f^{k+1}=p_{2}+Q_{2} P_{1} f^{k}
$$

It can be demonstrated that the sequence $\left\{f^{k}\right\}$ converges to $f$ in norm, and that the convergence is strictly monotonic [20]. Furthermore, under proper conditions on the two subsets $\mathscr{P}_{1}$ and $\mathscr{P}_{2}$, this iterative reconstruction algorithm can also be shown to be stable against moderately noisy data [20]. Equation (4) represents therefore the step of an effective iterative procedure for retrieving the unknown function $f$.

\section{APPLICATION TO PATTERN RECONSTRUC- TION FROM TRUNCATED MEASUREMENTS}

It is well known that the following simple relationship exists between the far-field pattern of an antenna and the Fourier-type PWS $\mathbf{f}\left(k_{x}, k_{y}\right)$ of the aperture field [11]

$$
\mathbf{E}(r, \theta, \phi)=\frac{j e^{-j k r}}{r} k \cos \theta \mathbf{f}(k \sin \theta \cos \phi, k \sin \theta \sin \phi)
$$

where $k$ is the wavenumber and an $e^{j \omega t}$ time dependence has been assumed. If the far-field pattern is derived from 


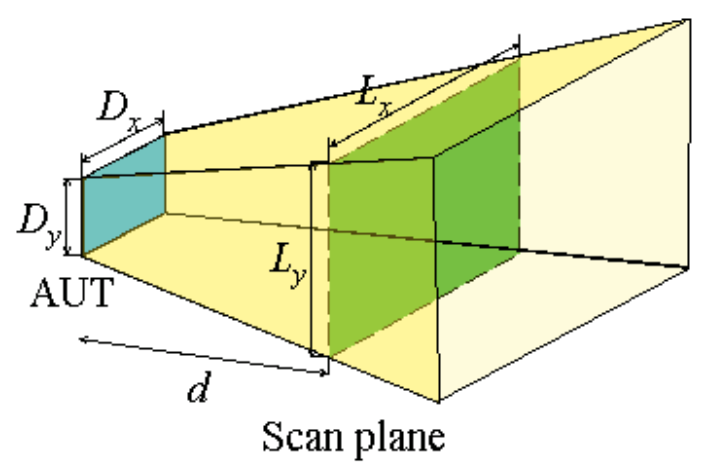

Figure 1. Geometry for the measurement setup and relevant angular reliable region for the reconstructed farfield pattern.

truncated planar near-field data, it can be assumed reliable only within the angular region defined by the rays running from the edge of the aperture through the boundary of the scan area [3] (Fig. 1). After defining the reliable angular region of the pattern on the basis of the AUT and scan plane geometry, the relationship (5) allows one to readily identify a reliable region of the PWS obtained by Fourier transforming the truncated spatial domain measurements. For instance, in the frequent case in which both the scan area and the AUT have a rectangular profile, centered with respect to the $z$ axis (Fig. 1), such a spectral region is defined by the following relationship

$$
\left\{\frac{k_{x}^{2}}{\left(k \sin \theta_{x}\right)^{2}}+\frac{k_{y}^{2}}{k^{2}}<1\right\} \cap\left\{\frac{k_{x}^{2}}{k^{2}}+\frac{k_{y}^{2}}{\left(k \sin \theta_{y}\right)^{2}}<1\right\}
$$

in which $\theta_{x}$ and $\theta_{y}$ are the reliable angles in the principal planes. These angles are defined by

$$
\theta_{x}=\frac{L_{x}-D_{x}}{2 d} ; \quad \theta_{y}=\frac{L_{y}-D_{y}}{2 d}
$$

where $D_{x}, D_{y}$ and $L_{x}, L_{y}$ are the dimensions of the AUT and of the scan plane along $x$ and $y$, respectively, and $d$ is the distance between the AUT and the scan plane. Equation (6) defines a spectral region contained within two ellypses, as shown in Fig. 2. After performing a probe correction in the spectral domain, like in [21], the spectrum is back propagated to the AUT plane; this step is readily performed through a near-field to near-field transformation [2]. Then, the problem of pattern reconstruction is rephrased in terms of extrapolation of the spectrum of a space-limited field and solved by applying the Gerchberg-Papoulis algorithm $[12,16]$, which constitutes a special implementation of the method of alternating orthogonal projections.

With respect to the notation introduced in the previous section, the subsets $\mathscr{P}_{1}$ can be identified with the subsets comprising all field distributions which vanish outside the antenna aperture, and $\mathscr{P}_{2}$ with the subset comprising all functions whose spectrum is zero outside the reliable spectral region. Accordingly, $\mathbf{p}_{2}$ corresponds to

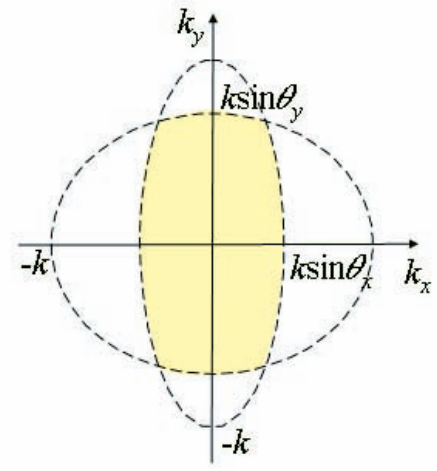

Figure 2. Reliable region of the Fourier aperture PWS for the measurement setup shown in Fig. 1 (yellow area).

the reliable part of the spectrum. Hence, following the algorithm described in the previous section, the iterative procedure consists of the following steps:

1. the spectrum is inverse Fourier transformed into the space domain to yield an estimate of the field distribution on the AUT plane;

2. the field distribution is projected onto the set $\mathscr{P}_{1}$, by setting all the samples outside the antenna aperture to zero;

3. thus modified, the field distribution is Fourier transformed back to the spectral domain ;

4. the generated spectrum is projected onto the set $\mathscr{Q}_{2}$ by setting to zero all the samples falling inside the reliable region;

5. the resulting pattern is added to the known projection $p_{2}$, so that the previous values are restored where the original spectrum is reliable.

These steps are iteratively applied until a proper convergence criterion (based on the estimated object energy outside the assumed extent of the aperture field or the difference function energy between the estimated and the true spectrum over the range of the known spectrum) is reached.

To sum up, the first step of the proposed procedure consists in deriving the PWS of the aperture field on the AUT plane aperture from the truncated measurements, i.e.

$$
\mathbf{f}^{0}\left(k_{x}, k_{y}\right)=\mathscr{F}\left\{\mathbf{E}_{S}(x, y) u_{S}(x, y)\right\} e^{j k_{z} d}
$$

where $\mathbf{E}_{S}$ is the scan plane tangential field, $\mathscr{F}$ is the Fourier transform operator, and $u_{S}(x, y)$ is a function equal to 1 for $(x, y)$ belonging to the scan area and equal to 0 elsewhere. In (8), probe correction has been neglected for the sake of simplicity. Then, the first iteration of the algorithm can be formulated as follows

$$
\begin{aligned}
& \mathbf{p}_{1}(x, y)=\mathscr{F}^{-1}\left\{\mathbf{f}^{0}\left(k_{x}, k_{y}\right)\right\} u(x, y) \\
& \mathbf{q}_{2}\left(k_{x}, k_{y}\right)=\mathscr{F}\left\{\mathbf{p}_{1}(x, y)\right\}\left[1-U_{R}\left(k_{x}, k_{y}\right)\right] \\
& \mathbf{f}\left(k_{x}, k_{y}\right)=\mathbf{f}^{0}\left(k_{x}, k_{y}\right) U_{R}\left(k_{x}, k_{y}\right)+\mathbf{q}_{2}\left(k_{x}, k_{y}\right) .
\end{aligned}
$$

where $u(x, y)$ is a function equal to 1 inside the AUT aperture area and to 0 elsewhere, and $U_{R}\left(k_{x}, k_{y}\right)$ is equal to 1 inside the reliable region and to 0 elsewhere. 
The generic iteration is then

$$
\begin{aligned}
& \mathbf{f}^{k+1}\left(k_{x}, k_{y}\right)=\mathbf{f}^{0}\left(k_{x}, k_{y}\right) U_{R}\left(k_{x}, k_{y}\right)+ \\
& \mathscr{F}\left\{\mathscr{F}^{-1}\left\{\mathbf{f}^{k}\left(k_{x}, k_{y}\right)\right\} u(x, y)\right\}\left[1-U_{R}\left(k_{x}, k_{y}\right)\right]
\end{aligned}
$$

This procedure converges to the true solution in the sense that the energy of the error spectrum, given by the difference between the correct spectrum and the reconstructed one, decreases monotonically for an increasing number of iterations. This can be explained as follows. The error spectrum is zero over the region in which the correct spectrum is known; as a consequence, its inverse Fourier transform has an infinite extent. This means that the spatial counterpart of the spectral error is distributed all over the infinite aperture plane. By setting to zero all the values outside the known extent of the true aperture, the algorithm will therefore reduce the energy of the spatial error, and, by Parseval's theorem [22], also the energy of the error spectrum. The error function so corrected has a spectral counterpart distributed all over the spectral plane. Thus, the further spectral manipulation, with substitution of zero spectral error in the reliable region, implies a further reduction of the error energy. Thus, at each iteration, the error energy is reduced twice. However, the amount of error energy reduction per iteration decreases by increasing the number of iterations.

The construction holds under the assumption that on the aperture plane the field distribution is zero outside the AUT boundary. This condition is rigorously satisfied in the presence of an infinite ground plane, while for finite apertures the presence of diffracted fields gives a minimum amount of energy outside the aperture. In these cases, a more general constraint, taking into account the expected behavior of the diffracted field, could be applied to increase the effectiveness of the method [23]. However, it has been found that an early termination of the iterations can yield a useful extrapolation of the spectrum of interest, provided that the energy of the unknown field distribution outside the assumed spatial extent is sufficiently small. This result has been also theoretically justified by Papoulis [16]. In practice, better results are obtained using in the procedure an equivalent aperture extending slightly beyond the AUT boundaries, to take into account the presence of diffracted fields, even if this will in general slow down the convergence of the algorithm. The algorithm is also subject to other sources of errors, such as discretization, noise, distortion and computer roundoff. These issues have been studied in [12] and [16] and have been shown to be a relatively minor problem. In particular, noise and distortion may to a certain extent be removed from the measured spectrum data on the basis that they are generally not capable of being continued to yield a finite object of a given extent.

The resulting procedure is very simple, only requiring the computation of Fourier transforms, which can be made quite rapid by the use of Fast Fourier Transform techniques [24]. As a matter of fact, the spectrum reconstructed from near-field data and back propagated to the AUT plane is typically restricted to the visible region. Hence, in practical implementation of the proposed algorithm, the spectrum is extended by zero filling before starting the iterative procedure. This step allows the it-

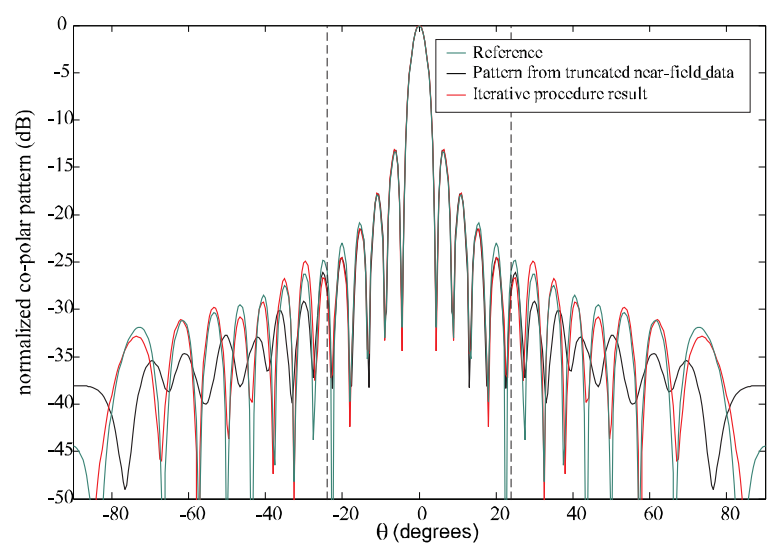

(a)

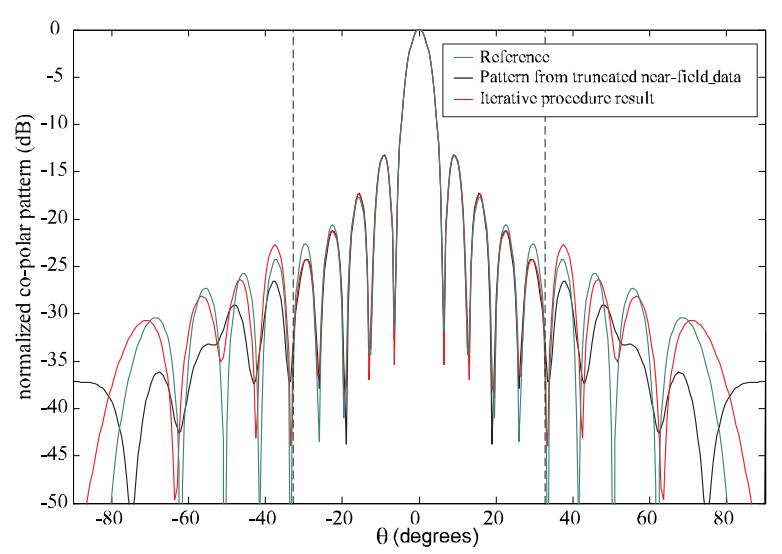

(b)

Figure 3. Comparison between the pattern obtained by the conventional near-field to far-field transformation of truncated simulated data and pattern obtained by applying the proposed procedure. The boundaries of the reliable region are shown with vertical dashed lines. a) uniform aperture, $\phi=0^{\circ}$ plane. $b$ ) electric dipole array, $\phi=0^{\circ}$ plane.

erative procedure result in a valid estimate also of the evanescent components of the PWS; such components, while negligible on the scan plane, are necessary to correctly represent the aperture field distribution.

The method of alternating orthogonal projection could in principle be directly applied to extrapolate the near-field data, by exploiting the condition that on the measurement plane the spectrum is limited to the visible region. However, preliminary investigations conducted on simulated data have shown a much slower convergence as compared to the proposed procedure; indeed, such an alternative approach does not take advantage of the a priori information on the antenna geometry and position. Furthermore, in practical cases the necessity of probe correcting the measured data makes it preferable performing the extrapolation process in the spectral domain. 


\section{NUMERICAL RESULTS}

The proposed procedure has first been validated through the application to noiseless simulated near-field data. The first case is a $3 \mathrm{~m} \times 3 \mathrm{~m}$ square aperture uniformly illuminated with a $x$-polarized electric field at the frequency of $1.307 \mathrm{GHz}$, and thus a wavelength of about $0.23 \mathrm{~m} .35 \times 35$ near-field samples separated by a half wavelength distance are collected in a plane at a distance of $5 \lambda$ from the antenna aperture. Thus, the scan plane dimensions are $L_{x}=L_{y} \simeq 4 \mathrm{~m}$, corresponding to the reliability angles $\theta_{x}=\theta_{y} \simeq 24^{\circ}$. In Fig. 3(a), the amplitude of the co-polar component of the radiated field (according to Ludwig's third definition [25]) obtained by directly applying a near-field to far-field transformation to this truncated set of data is compared with the results obtained after 25 iterations of the proposed iterative technique; the theoretical pattern is also shown as a reference. It is apparent that a very good estimate of the pattern is obtained also for the wide angle lobes. It is wort noting that the fact that the near-field data are noiseless does not imply that the part of the spectrum which is assumed reliable is error free. Indeed, the scan plane truncation causes some limited errors also inside the so called reliable region [3]. As a consequence, the good results obtained for this first configuration testify the validity of the proposed procedure when applied to moderately noisy data.

As a second test case, a $12 \times 12$ element array of $x$ polarized half-wavelength electric dipoles has been considered. The operating frequency is $1.51 \mathrm{GHz}$, corresponding to a wavelength of about $0.2 \mathrm{~m}$. The distances between the elements along $x$ and $y$ are $d_{x}=d_{y}=0.15$ $\mathrm{m}$; thus, the dimensions of the array are $D_{x}=D_{y}=1.8$ m. $31 \times 31$ near-field samples separated by a half wavelength distance are collected in a plane at a distance of $5 \lambda$ from the antenna aperture. Thus, the scan plane dimensions are $L_{x}=L_{y} \simeq 3 \mathrm{~m}$, corresponding to the reliability angles $\theta_{x}=\theta_{y} \simeq 33^{\circ}$. The results obtained for to the co-polar component of the radiated field in the $\phi=0^{\circ}$ plane by applying 10 iterations of the proposed procedure are shown in Fig. 3(b). Although in this case, differently from the previous configuration, the electric field on the AUT plane is not zero outside the AUT boundaries, numerical results show that the proposed procedure can anyway provide a useful correction to the far-field pattern with a quite small number of iterations. However, it must be noted that the improvement is less significant in the orthogonal plane.

The iterative algorithm has been also applied to measured near-field data and some results relevant to a $x$-polarized rectangular horn antenna are reported in Fig. 4. The antenna dimensions along $x$ and $y$ are $D_{x}=161 \mathrm{~mm}$ and $D_{y}=226 \mathrm{~mm}$, respectively, but larger values $\left(D_{x}=211\right.$ $\mathrm{mm}, D_{y}=253 \mathrm{~mm}$ ) have been considered in the iterative procedure in order to account for the presence of the diffracted field; the near-field was measured at $13 \times 13$ points, covering an area of dimensions $L_{x}=L_{y}=325$ $\mathrm{mm}$. The distance between the scan plane and the AUT was $d=70 \mathrm{~mm}$, corresponding to the reliability angles $\theta_{x} \simeq 39^{\circ}$ and $\theta_{y} \simeq 27^{\circ}$. The operating frequency was $5.3 \mathrm{GHz}$. Fig. 4(a) reports the results for the co-polar pattern in the plane $\phi=0^{\circ}$, while the results relevant to the

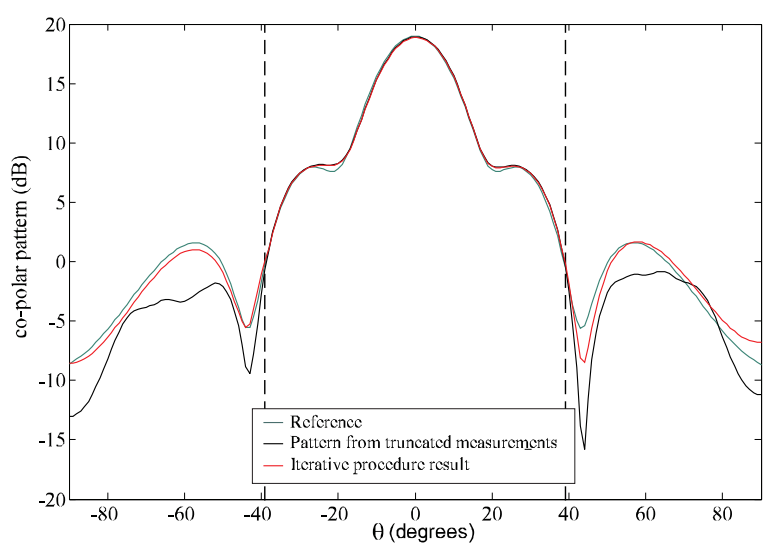

(a)

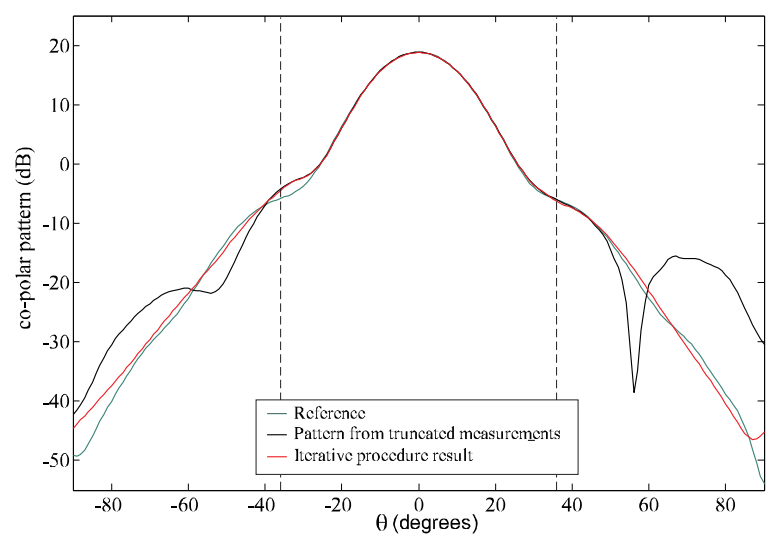

(b)

Figure 4. Far-field pattern from a rectangular horn: comparison between the pattern obtained by the conventional near-field to far-field transformation of truncated measured data and pattern obtained by applying 60 iterations of the proposed procedure. The boundaries of the reliable region are shown with vertical dashed lines. a) $\phi=0^{\circ}$ plane. b) $\phi=135^{\circ}$ plane.

plane $\phi=135^{\circ}$ are shown in Fig. 4(b). The reference pattern has been obtained by independent measurements in the DTU-ESA facility. Also in this case, a very good far-field pattern estimate is obtained.

\section{CONCLUSIONS}

In this work, an effective application of the alternating projections algorithm is proposed for truncation error reduction; it exploits the a priori knowledge on the AUT geometry and position and it exhibits a quite fast convergence. The procedure is based on the use of the reliable part of the spectrum and on the requirement that the tangential electric field is concentrated on the antenna aperture. The algorithm has been applied to simulated as well as measured near-field data and in both cases it has turned out to be very effective. 


\section{REFERENCES}

1. R. C. Johnson, H. Allen Ecker, and J. S. Hollis. Determination of far-field antenna patterns from near-field measuremets. Proc. IEEE.

2. J. J. H. Wang. An examination of the theory and practice of planar near-field measurement. IEEE Trans. Antennas Propagat., 36:746-753, 1988.

3. A. D. Yaghjian. Upper bound errors in far-field antenna parameters determined from planar near-field measurements. Technical Report NBS Tech. Notes 667, Nat. Bur. Stand., Boulder, CO, Hamburg, 1975.

4. E. B. Joy. Windows 96 for planar near-field measurements. In AMTA Proceedings, pages 80-85, 1996.

5. P. R. Rousseau. The development of a near-field data window function for measuring standard gain horns. In AMTA Proceedings, pages 96-101, 1997.

6. P. R. Rousseau. The planar near-field measurement of the far sidelobes of a reflector antenna. In IEEE AP-S Symposium Digest, pages 1315-1318, 1998.

7. P. Petre and T. K. Sarkar. Planar near-field to farfield transformation using an equivalent magnetic current approach. IEEE Trans. Antennas Propagat., 40:1348-1356, Nov. 1992.

8. J.R. Perez and J. Baserrechea. Analysis of different GA strategies applied to antenna far-fields reconstruction from planar acquisition. Microw. Opt. Tech. Lett., 39:422-426, 2003.

9. O. M. Bucci, G. D’Elia, and M. D. Migliore. A new strategy to reduce truncation error in near-field/farfield transformations. Radio Science, 35:3-17, 1973.

10. S. M. Vovk and V. F. Borulko. Using the method of minimum extension to extrapolation of antenna measurements. In 10th Int. Conf. on Mathematical Methods in Electromagnetic Theory, Dniepropetrovsk, Ukraine.

11. H. G. Booker and P. C. Clemmow. The concept of an angular spectrum of plane waves, and its relations to that of polar diagram and aperture distribution. Proc. Inst. Elec. Eng.

12. R. W. Gerchberg. Super-resolution through error energy reduction. Optical Acta, 21(9):709-720, 1974.

13. D. Slepian and H. O. Pollak. Prolate spheroidal wave functions, Fourier analysis and uncertainty - I. Bell Syst. Tech. J., 40:43-84, Jan. 1961.

14. C. W. Barnes. Object restoration in a diffractionlimited imaging system. J. Opt. Soc. Am., 56(5):575$578,1966$.

15. C. K. Rushforth and R. W. Harris. Restoration, resolution and noise. J. Opt. Soc. Am., 58(4):539-545, 1968.
16. A. Papoulis. A new algorithm in spectral analysis and band-limited extrapolation. IEEE Trans. Circuits Syst., CAS-22:735-742, 1975.

17. Y. Rahmat-Samii. Surface diagnosis of large reflector antennas using microwave holographic metrology: An iterative approach. Radio Science, 19(5):1205-1217, 1984.

18. M. S. Narasimhan and B. Preetham Kumar. A technique of synthesizing the excitation currents of planar arrays or apertures. IEEE Trans. Antennas Propagat., 38(9):1326-1332, 1990.

19. M. Johansson and B. Svensson. Array antenna diagnosis and calibration. In AMTA Proceedings, pages 10-27-10-32, 1990.

20. D. C. Youla. Generalized image restoration by the method of alternating orthogonal projections. IEEE Trans. Circuits Syst., CAS-25:694-702, 1978.

21. T. B. Hansen and A. D. Yaghjian. Plane-wave theory of time domain fields. Wiley-IEEE Press, 1999.

22. A. Papoulis. The Fourier integral and its applications. McGraw-Hill, New York, 1962.

23. H. Peng and H. Stark. Signal recovery with similarity constraints. J. Opt. Soc. Am. A, 6(6).

24. J. W. Cooley, P. A. W. Lewis, and P. D. Welch. Application of the Fast Fourier Transform to computation of Fourier integrals, Fourier series, and convolution integrals. IEEE Trans. Audio Electroacustics, AU15:79-84, 1967.

25. A. C. Ludwig. The definition of cross-polarization. IEEE Trans. Antennas Propagat., AP-21:116-119, 1973. 